\title{
Acute necrotizing pneumonia combined with parapneumonic effusion caused by Mycobacterium lentiflavum: a case report
}

\author{
Yong Chul Lee ${ }^{*+}$, Seung Bum Kim, Su Jin Gang, Seung Yong Park and So Ri Kim ${ }^{*+}$
}

\begin{abstract}
Background: Mycobacterium lentiflavum (M. lentiflavum), a slow growing nontuberculous mycobacterium (NTM), has recently been described as an emerging human pathogen regardless of the immune status of the host. Previous reports have demonstrated that cervical lymphadenitis of children is the most frequent pathology of $M$. lentiflavum. However, there are little reports regarding pulmonary diseases by M. lentiflavum specifically in immunocompetent patients.

Case presentation: A 60-year-old man having prolonged productive cough and dyspnea with fever was initially diagnosed as pneumonia with parapneumonic effusion. Imaging studies showed that the radiologic abnormality was acute bronchopneumonic infiltration with abscess formation in the left lower lobe and parapneumonic pleural effusion. M. lentiflavum was identified in the cultured pleural tissues. On the basis of these findings, he was diagnosed as pulmonary infection and pleurisy caused by M. lentiflavum, which was treated with a combination of antibiotics covering NTM. His clinical manifestations were dramatically improved by the treatment targeting NTM, while those were refractory to empirical antibiotic therapy.

Conclusion: In this report, we introduce the isolation of $M$. lentiflavum from pleural tissues associated with acute necrotizing pneumonia combined with parapneumonic effusion in an immunocompetent host, suggesting that the M. lentiflavum can be a human pathogen invovled in pulmonary infectious diseases and pleurisy with poor response to empirical antibiotic treatment.
\end{abstract}

Keywords: Mycobacterium lentiflavum, Nontuberculous mycobacterium, Pleural effusion, Necrotizing pneumonia

\section{Background}

Mycobacterium lentiflavum (M. lentiflavum), one of slowly growing nontuberculous mycobacterium (NTM), was first identified in 1996 as a distinct strain [1]. This strain is characterized by slow growth of tiny yellow colonies and has biochemical characteristics identical to the Mycobacterium avium complex (MAC). In addition, mycolic acid and fatty acid chromatography patterns are very similar to those of Mycobacterium simiae [1]. Thus, the identification of $M$. lentiflavum is usually confirmed

\footnotetext{
* Correspondence: leeyc@jbnu.ac.kr; sori@jbnu.ac.kr

${ }^{\dagger}$ Equal contributors

Department of Internal Medicine, Research Center for Pulmonary Disorders, Chonbuk National University Medical School, Research Institute of Clinical Medicine of Chonbuk National University-Biomedical Research Institute of Chonbuk National University Hospital, Jeonju, South Korea
}

through genetic analysis. Because like other NTM, $M$. lentiflavum is often isolated fortuitously, the clinical significance must be carefully considered.

Recently several cases of human diseases caused by $M$. lentiflavum have been reported, including cervical lymphadenitis, fatal disseminated infection, ascites, soft tissue, liver abscess, and pulmonary infection in immunocompetent and immunosuppressed patients [2-12]. However, there are few reports on $M$. lentiflavum as a cause of pulmonary disease and to date it has rarely been described in immunocompetent patients.

Herein, we present a case of acute necrotizing pneumonia combined with parapneumonic effusion caused by $M$. lentiflavum in an immunocompetent patient.

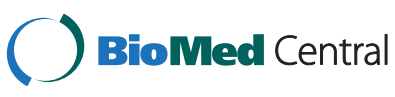

(c) 2015 Lee et al. Open Access This article is distributed under the terms of the Creative Commons Attribution 4.0 International License (http://creativecommons.org/licenses/by/4.0/, which permits unrestricted use, distribution, and reproduction in any medium, provided you give appropriate credit to the original author(s) and the source, provide a link to the Creative Commons license, and indicate if changes were made. The Creative Commons Public Domain Dedication waiver (http://creativecommons.org/publicdomain/zero/1.0/) applies to the data made available in this article, unless otherwise stated. 


\section{Case presentation}

A 60-year-old man was admitted to our hospital with fever, chills, productive cough, and dyspnea for last two weeks, which had exacerbated for preceding three days despite taking oral antibiotics for about 10 days. Since he had been diagnosed to have bronchial asthma three years ago, he had taken the combined inhaled medicine of corticosteroid and a long-acting $\beta_{2}$ agonist. However, he had no previous history of structural lung diseases including pulmonary tuberculosis and immunocompromised disorders such as HIV infection. He was an ex-smoker with 20 pack/year smoking history and his occupation was a farmer. On physical examination, inspiratory crackles were heard mainly on the left middle and lower lung fields. Chest radiography showed pulmonary consolidation with air bronchogram in the left lower lobe, which also showed fluid shifting sign (Fig. 1a and b). Chest computed tomography (CT) scan revealed bronchopneumonic infiltration with abscess formation in the left lower lobe, accompanying left pleural effusion (Fig. 1c and d). Bacteriologic examinations including Gram stain with sputum, pleural fluids, and bronchial washing fluids revealed no definitive causative organisms. Pleural tissues were also obtained through needle biopsy, in which the acute suppurative pleural inflammatory reaction was found and some tissues were cultured to examine any bacterial or fungal growth. As a result, there was no

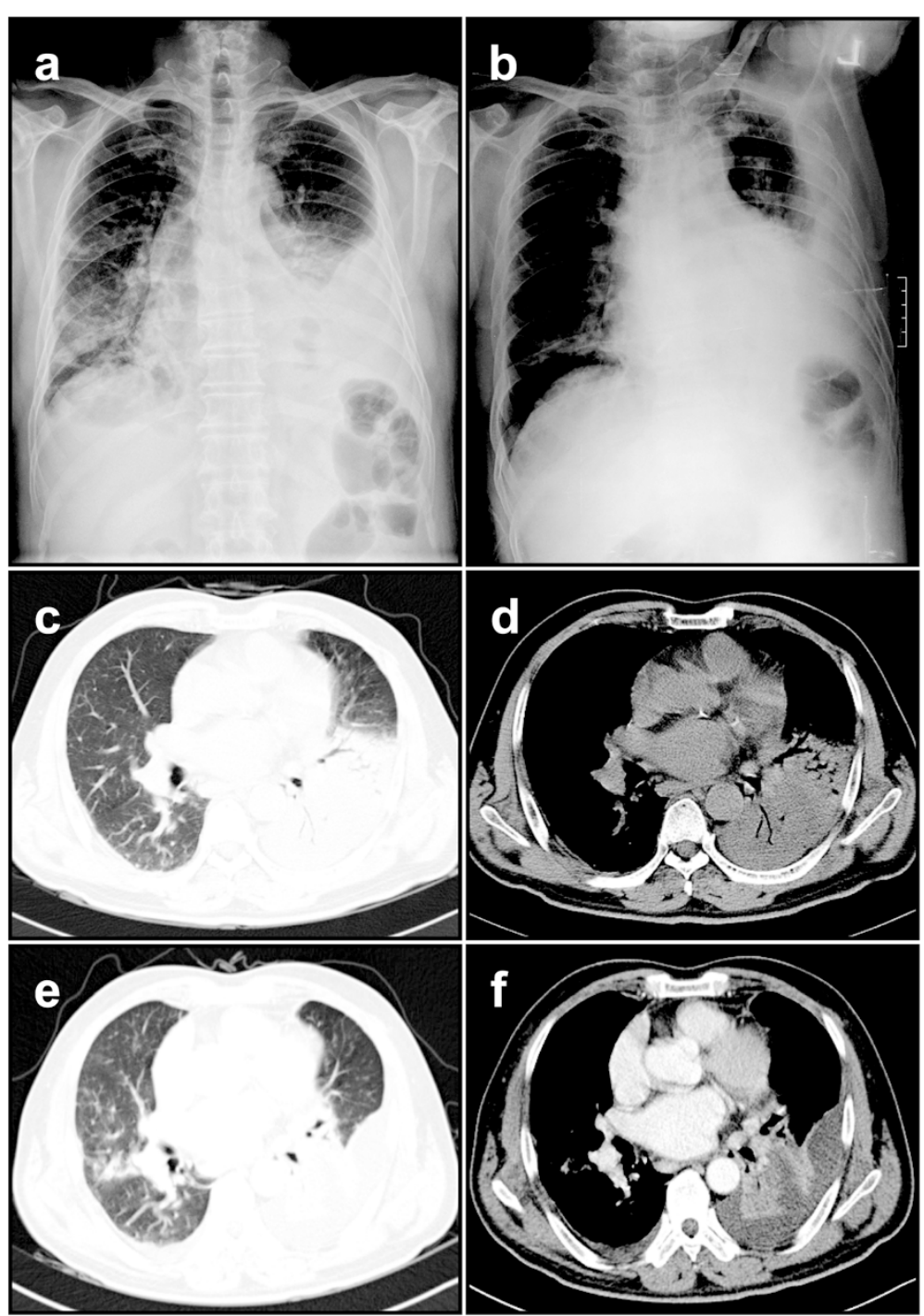

Fig. 1 Chest X-ray and chest computed tomography (CT) findings in M. lentiflavum-infected patient. Chest Radiography (a; posteroanterior view, b; left decubitus view) shows consolidative lesion in the left lower lobe with fluid shifting sign. Chest CT scan revealed that solid consolidative lesion occupied the left lower lobe with air-bronchogram (c and $\mathbf{d}$ ). Despite of the antibiotics therapy, the radiologic features were aggravated showing abscess formation in the left lower lobe, accompanying left pleural effusion and newly developed pneumonic infiltrative lesion on the right middle lobe (e and $\mathbf{f}$ ) 
evidence of growth of bacteria or fungi for seven days they had been cultured.

Firstly, levofloxacin was prescribed as a single intravenous regimen. Up to hospital day 3, however, his clinical manifestation did not respond to the antibiotic therapy and even worsened (Fig. 1e and f). We decided to change the antibiotic regimen to aztreonam and metronidazole as a combination regimen. On hospital day 7 , his symptoms including fever started to be resolved and then followed by radiologic improvement on chest radiography. After his discharge, we got a final bacteriologic report describing that M. lentiflavum was isolated from pleural specimen biopsied. The antibiotic regimen covering NTM (etambutol, ciprofloxacin, rifabutin, and clarithromycin) was prescribed to him in out-patient department, and his remnant lesions kept being improved (Fig. 2a-c), showing bronchiectasis and multiple smaller nodules of a tree-in-bud pattern in the left lingular segment and the left lower lobe (Fig. $2 \mathrm{~d}$ and e).

\section{Discussion}

In 1959, Runyon proposed the first classification system of mycobacteria in which, based on growth rates, colony morphology, and pigmentation in the presence and absence of light, mycobacteria were divided into four groups of human pathogens; $M$. tuberculosis complex, $M$. leprae, slowly growing NTM, and rapidly growing NTM [13]. The NTM are usually acquired from environmental sources such as surface water, tap water, soil, domestic and wild animals, milk, and food products. Though most species are less pathogenic or sporadic, they can, in broad terms, induce four distinct clinical syndromes [14]; 1) Progressive pulmonary disease, especially in older persons with or without underlying lung disease. 2) Superficial lymphadenitis, especially cervical lymphadenitis. 3) Disseminated disease in severely immunocompromised patients. 4) Skin and soft tissue infection usually as a consequence of direct inoculation.

Recently, with the availability of $16 \mathrm{~S}$ ribosomal RNA sequencing and high-performance liquid chromatography, and polymerase chain reaction-restriction length polymorphism analysis, the number of new species of NTM including $M$. lentiflavum has risen dramatically $[14,15]$. In fact, $M$. lentiflavum, one of slowly growing NTM, was first identified in a cluster of 22 isolates [1]. Among them, only one isolate from a vertebral disk in an elderly patient suffering from spondilodiscitis was clinically significant. Since then, several cases of isolates from cervical lymphadenitis of very young children have been reported [2-8]. Other infected sites including pulmonary infection are less frequent $[1,4,6-12]$.

Typically, pulmonary disease by NTM is characterized as chronic progressive infectious one which develops both in healthy persons and in those with underlying pulmonary disorders or immunosuppressive conditions. The clinical features may resemble slowly progressive pulmonary tuberculosis, which is often the initial diagnosis in patients with positive results on acid fast bacilli (AFB) staining. Radiologic manifestations of pulmonary infection with NTM are various, to some degree, non-specific including the formation of solitary or multiple nodules, chronic pneumonitis,

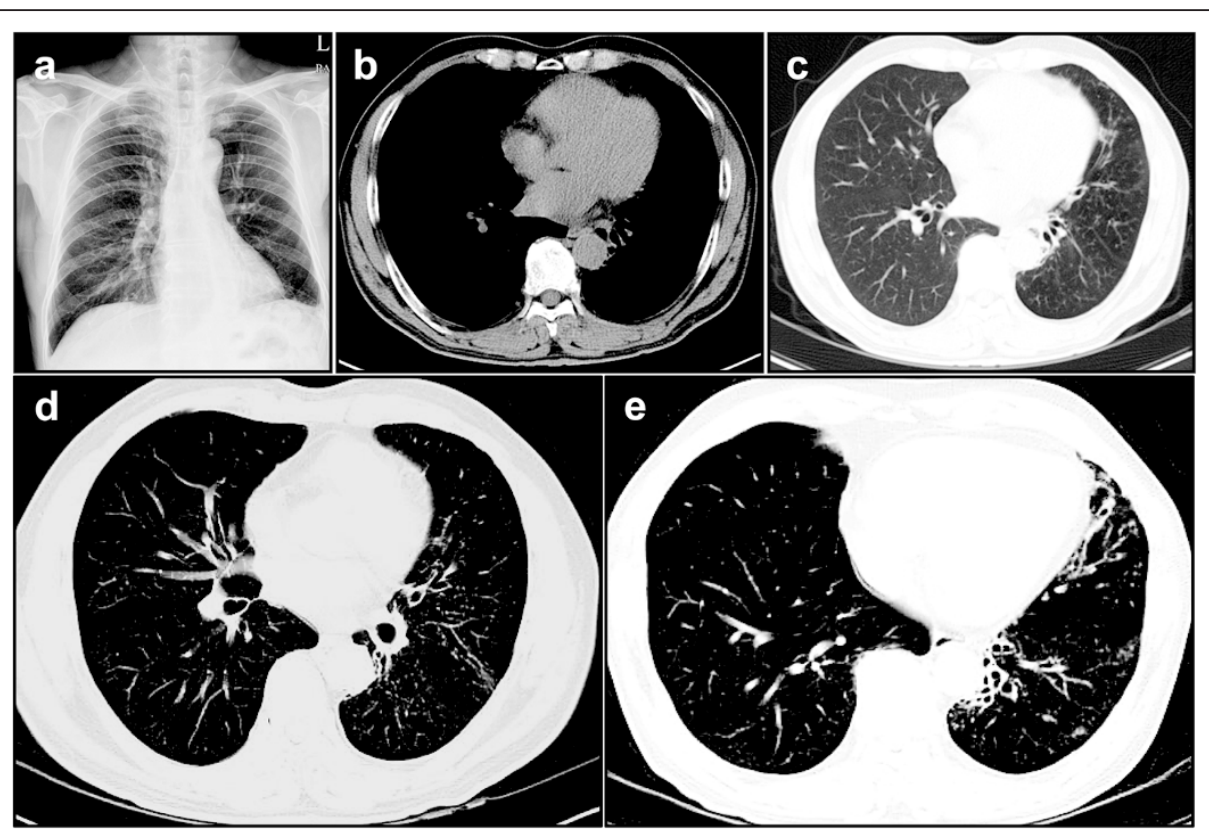

Fig. 2 Serial chest imaging findings in M. lentiflavum-infected patient. At 12 month follow-up, chest imaging studies revealed that the previous lesions in both lungs were improved remarkably (a-c). In addition, serial chest CT scans showed the distinct bronchiectasis and multiple small nodular lesions with a tree-in-bud pattern were scattered in the left lingular segment and the left lower lobe (d and e) 
bronchiectasis, cavitary formation, or a combination of these features.

Altogether, the diagnosis of pulmonary infection with NTM is extremely difficult due to several factors involved; these are various clinical and radiologic manifestations, the frequent presence of significant prior pulmonary disease, and harmless colonization of NTM in the lower respiratory tracts. According to American Thoracic Society (ATS)/ Infectious Diseases Society of America (IDSA) guidelines, the diagnosis should be based on specific, validated criteria that emphasize a compatible clinical syndrome, characteristic findings on chest X-ray or $\mathrm{CT}$, and repeated isolation of NTM from the sputum or growth of NTM from a lung biopsy [16]. Thus, in cases with these typical radiologic findings but with negative results of routine sputum cultures for mycobacteria, physicians have been subsequently recommended to perform bronchoscopy and transbronchial biopsy.

To our knowledge, this report is the first case of acute necrotizing pneumonia with pleurisy by $M$. lentiflavum in an immunocompetent patient without underlying pulmonary structural disorders. In our case, a 60-year-old man having prolonged productive cough and dyspnea with fever was initially diagnosed as pneumonia with parapneumonic effusion. Imaging studies showed that the radiologic abnormality was acute bronchopneumonic infiltration with abscess formation in the left lower lobe and parapneumonic pleural effusion. Bacteriologic examinations with sputum, pleural fluids, and bronchial washing fluids, however, revealed no definitive causative organisms. Fortunately, we could confirm that the etiologic organism was $M$. lentiflavum found in the cultured pleural tissues, although there is still a possibility of co-infection with other bacteria such as anaerobes because it is difficult to culture or isolate anaerobes in several respiratory samples and the patient was pre-treated with the antibiotics that would have negated bacterial cultures or have activity against co-infected bacteria. On the basis of these findings, he was diagnosed as pulmonary infection and pleurisy caused by M. Lentiflavum as at least one of etiological pathogens, which was treated with a combination of antibiotics covering NTM.

\section{Conclusions}

In light of general information of NTM, this case report describes several interesting points as follows; the acute progress of NTM pulmonary diseases, pleural disease by NTM infection, and occurence of pulmonary infection by M. lentiflavum in immunocompetent subject. Thus, we suggest that clinicians should consider the M. lentiflavum as a human pathogen invovled in lung and pleura regardless of immune status. In addition, $M$. lentiflavum can be an infectious cause of acute pulmonary disease as well as chronic pulmonary disease.

\section{Consent}

Written informed consent was obtained from the patient for publication of this case report. Copies of the written consents are available for review by the editor of this journal.

\section{Abbreviations}

MAC: Mycobacterium avium complex; NTM: Nontuberculous mycobacterium.

\section{Competing interests}

The authors declare that they have no competing interests.

\section{Authors' contributions}

YCL and SRK designed the study. YCL and SRK drafted the manuscript. YCL, SRK, SBK, and SYP contributed to the diagnosis and treatment. SBK contributed to the analysis and identification of M. lentiflavum. SJG reviewed and edited the manuscript. YCL and SRK reviewed and supervised the manuscript. All the authors approved the final version of the manuscript.

\section{Acknowledgments}

We thank Professor Mie-Jae Im (Chonbuk National University Medical School, Jeonju, South Korea) for critical readings of the manuscript. This study was supported by the Korea Healthcare Technology R\&D Project, Ministry for Health and Welfare, Republic of Korea (Grant A121931), by Basic Science Research Program through the National Research Foundation of Korea (NRF) funded by the Ministry of Science, ICT and future Planning (NRF-

2014R1A2A1A01002823), and by the fund of Biomedical Research Institute, Chonbuk National University Hospital.

Received: 20 January 2015 Accepted: 10 August 2015

Published online: 19 August 2015

\section{References}

1. Springer B, Wu WK, Bodmer T, Haase G, Pfyffer GE, Kroppenstedt RM, et al. Isolation and characterization of a unique group of slowly growing mycobacteria: description of Mycobacterium lentiflavum sp. nov. J Clin Microbiol. 1996;34:1100-7.

2. Cabria F, Torres MV, Garcia-Cia Jl, Dominguez-Garrido MN, Esteban J, Jimenez MS. Cervical lymphadenitis caused by Mycobacterium lentiflavum. Pediatr Infect Dis. 2002;21:574-5.

3. Haase G, Kentrup H, Skopnik H, Springer B, Böttger C. Mycobacterium lentiflavum: an etiological agent of cervical lymphadenitis. Clin Infect Dis. 1997;25:1245-6.

4. Tortoli E, Bartoloni A, Erba ML, Levrè E, Lombardi N, Mantella A, et al. Human infections due to Mycobacterium lentiflavum. J Clin Microbiol. 2002;40:728-9.

5. Uria MJ, Garcia J, Menendez JJ, Jimenez MS. Mycobacterium lentiflavum infection: case history and review of the medical literature. Enferm Infecc Microbiol Clin. 2003;21:274-5.

6. Ibanez R, Serrano-Heranz R, Jimenez-Palop M, Roman C, Corteguera M, Jimenez S. Disseminated infection caused by slowgrowing Mycobacterium lentiflavum. Eur J Clin Microbiol Infect Dis. 2002;21:691-2.

7. Niobe SN, Bebear CM, Clerc M, Pellegrin JL, Bebear C, Maugein J. Disseminated Mycobacterium lentiflavum infection in a human immunodeficiency virus-infected patient. J Clin Microbiol. 2001;39:2030-2.

8. Galarraga MC, Torreblanca A, Jimenez MS. Isolation of Mycobacterium lentiflavum in a case of suspected lung cancer. Enferm Infecc Microbiol Clin. 2002;20:93-4.

9. Molteni C, Gazzola L, Cesari M, Lombardi A, Salerno F, Tortoli E, et al. Mycobacterium lentiflavum infection in immunocompetent patient. Emerg Infect Dis. 2005;11:119-22.

10. Tortoli E, Mattei R, Russo C, Scarparo C. Mycobacterium lentiflavum, an emerging pathogen? J Infect. 2006;52:185-7.

11. Shin S, Yoon JH, Song SH, Kim EC. Isolation of Mycobacterium lentiflavum from a patient with a lung destroyed by tuberculosis. Korean J Lab Med. 2007;27:124-7.

12. Shamaei M, Marjani M, Farnia P, Tabarsi P, Mansouri D. Human infections due to Mycobacterium lentiflavum; first report in Iran. IRAN J Microbiol. 2010;2:29-31. 
13. Runyon EH. Anonymous mycobacteria in pulmonary disease. Med Clin North Am. 1959:43:273-90.

14. Wolinsky E. Nontuberculous mycobacteria and associated diseases. Am Rev Respir Dis. 1979;119:107-59.

15. Brown-Elliott BA, Griffith DE, Wallace Jr RJ. Newly described or emerging human species of nontuverculous mycobacteria. Infect Dis Clin North Am. 2002;16:187-220

16. Griffith DE, Aksamit T, Brown-Elliott BA, Catanzaro A, Daley C, Gordin F, et al. ATS Mycobacterial Diseases Subcommittee; American Thoracic Society; Infectious Disease Society of America. An official ATS/IDSA statement: diagnosis, treatment, and prevention of nontuberculous mycobacterial disease. Am J Respir Crit Care Med. 2007;175:367-416.

\section{Submit your next manuscript to BioMed Central} and take full advantage of:

- Convenient online submission

- Thorough peer review

- No space constraints or color figure charges

- Immediate publication on acceptance

- Inclusion in PubMed, CAS, Scopus and Google Scholar

- Research which is freely available for redistribution 